\begin{abstract}
Nr. $215 / 1996$
Stability of Solutions of PDE's With

RANDOM DRIFT AND VISCOSITY LIMIT

Th. Deck, J. Potthoff, G. Våge, H. Watanabe
\end{abstract}




\title{
Stability of Solutions of PaRabolic PDE's WITH RANDOM DRIFT AND VISCOSITY LIMIT
}

\author{
T. Deck ${ }^{1, a}$, J. Potthoff ${ }^{1, a}$, G. Vage ${ }^{1, b}$ and H. Watanabe ${ }^{2}$ \\ 1 University of Mannheim, D-68131 Mannheim, Germany. \\ ${ }^{2}$ Department of Applied Mathematics, Faculty of Science, \\ Okayama University of Science, Japan.
}

\begin{abstract}
Let $u_{\alpha}$ be the solution of the Itô stochastic parabolic Cauchy problem $\partial u / \partial t-L_{\alpha} u=\xi \cdot \nabla u,\left.u\right|_{t=0}=f$. We prove that $u_{\alpha}$ depends continuously on $\alpha$, when the coefficients in $L_{\alpha}$ converge to those in $L_{0}$. This result is used to study the diffusion limit for the Cauchy problem (in Stratonovich sense): when the coefficients of $L_{\alpha}$ tends to 0 the corresponding solutions $u_{\alpha}$ converge to a function $u_{0}$ satisfying $\partial u_{0} / \partial t=\xi \circ \nabla u_{0},\left.u\right|_{t=0}=f$. A criterion is provided for the existence of strong limits, e.g. $u_{\alpha} \rightarrow u_{0}$, in the space of Hida distributions $(\mathcal{S})^{*}$. As an application we show that weak solutions of the above Cauchy problem are strong solutions.
\end{abstract}

\section{Introduction}

Consider an incompressible fluid with velocity field $w(t, x)=\left(w_{1}(t, x), w_{2}(t, x), w_{3}(t, x)\right)$ at time $t \in \mathbb{R}_{+}$and position $x \in \mathbb{R}^{3}$. The mass density $u(t, x)$ of particles suspended in this fluid satisfies the equation

$$
\frac{\partial u}{\partial t}-L u=-w \cdot \nabla u,\left.u\right|_{t=0}=f
$$

where $L u=\nabla(c \nabla u)$ (derivatives with respect to $\left.x \in \mathbb{R}^{3}\right), c(t, x)$ is the diffusion coefficient, and $f(x)$ the initial density of the particles. In [Ch], P.-L. Chow proposed the stochastic partial differential equation (1.1) with $w(t, x)=\dot{\eta}(t, x)$, where $\dot{\eta}_{i}(t, x)$ is the formal time derivative of $\eta_{i}(t, x)=\sum_{j=1}^{3} \int_{0}^{t} \sigma_{i j}(s, x) d B_{j}(s)$ for $i=1,2,3$, as a model for transport of particles in a turbulent medium. For the one-dimensional case he proved existence and uniqueness of a solution $u$ for this equation. Moreover, he observed that $u$ under natural conditions is a generalized random field. This motivated $[P],[D P]$ to consider the equation using white noise analysis. Applying contraction methods they proved existence and uniqueness of a weak solution of (1.1) for uniformly elliptic operators $L(t, x), x \in \mathbb{R}^{d}$, of order two when $w(t, x)=\dot{\eta}(t, x)$ is as above. (1.1) was recently considered again in [PVW]. Applying the Girsanov formula the authors were able to prove existence and uniqueness of a weak solution of (1.1) when $w$ is $d$-dimensional space-time white noise.

${ }^{a}$ Partially supported by the DFG.

${ }^{b}$ Supported by the DFG. 
In practice the molecular diffusion $c$ is small, i.e., $0<c \ll 1$. If we assume that $c=\alpha$ is constant, we are led to consider (1.1) with $L_{\alpha}=\alpha \Delta$, where $0<\alpha \ll 1$. What happens when $\alpha$ tends to 0 ? P.-L. Chow answers this question in [Ch], when $w(t, x)=\dot{\eta}(t, x)$, $x \in \mathbb{R}$. In this paper we consider the related problem of stability of the solution of (1.1) with respect to perturbations of the differential operator $L$ for more general types of noise. In particular, if $L_{\alpha}$ for $\alpha \in[0,1]$ is a family of differential operators such that the coefficients of $L_{\alpha}$ tend uniformly to the coefficients of $L_{0}$ as $\alpha \rightarrow 0$, then we provide conditions under which the corresponding solutions $u_{\alpha}$ converge to $u_{0}$. Moreover, we show that if $(1.1)$ is interpreted in Stratonovich sense and $L_{\alpha}$ tends uniformly to 0 , the corresponding solutions $u_{\alpha}$ converge to $u_{0}$, where $u_{0}$ solves the (degenerate) equation obtained in the limit. If (1.1) is interpreted in Itô sense the same result does not hold; one can actually construct explicit counterexamples.

The paper is organized as follows: In Section 2 we introduce basic notations. Section 3 contains the stability result. In Section 4 we consider the diffusion limit problem for (1.1) when the equation is interpreted in the Stratonovich sense. A crucial lemma needed to prove the stability theorem in Section 3 is given in the appendix. As a corollary to this lemma we obtain conditions under which a weak solution of (1.1) is a strong solution, in the sense that the derivatives exist in the strong sense. Since the solutions constructed in [PVW] satisfy these conditions, it follows that they are strong solutions.

\section{Notations and preliminaries}

Let $\mathcal{S}\left(\mathbb{R}^{d+1}\right)$ be the space of rapidly decreasing functions and $\left(\mathcal{S}^{\prime}\left(\mathbb{R}^{d+1}\right), \mathcal{B}, \mu\right)$ be the white noise probability space. It is well-known that there is a chain of Hilbert spaces $(\mathcal{S})_{q}$ with inner product $(\cdot, \cdot)_{q}, q \in \mathbb{Z}$, such that

$$
(\mathcal{S})^{*}=\bigcup_{q \in \mathbb{N}}(\mathcal{S})_{-q} \supset \cdots \supset(\mathcal{S})_{-1} \supset L^{2}(\mu) \supset(\mathcal{S})_{1} \supset \cdots \supset \bigcap_{q \in \mathbb{N}}(\mathcal{S})_{q}=(\mathcal{S})
$$

$(\mathcal{S})$ and $(\mathcal{S})^{*}$ denote the space of Hida test functions and of Hida distributions, respectively. We equip $(\mathcal{S})^{*}$ with the strong topology (which coincides with the inductive limit topology), and denote the dual pairing between $(\mathcal{S})$ and $(\mathcal{S})^{*}$ by $\langle\cdot, \cdot\rangle$. For $T>0$ and $d \in \mathbb{N}$ we set $D_{T}=\left\{(t, x) \mid t \in[0, T], x \in \mathbb{R}^{d}\right\}$. By a (generalized) random field $u \in C^{1,2}\left(D_{T}, \mathcal{S}^{*}\right)$ we mean a mapping $u: D_{T} \rightarrow(\mathcal{S})^{*}$ which is continuously differentiable in $t$ and twice continuously differentiable in $x$, in the strong sense. This means, e.g., that the limit

$$
\frac{\partial u}{\partial t}(t, x):=\lim _{\epsilon \rightarrow 0} \frac{u(t+\epsilon, x)-u(t, x)}{\epsilon}
$$

exists in $(\mathcal{S})^{*}$ and depends continuously on $(t, x) \in D_{T}$. In what follows we denote by $\dot{B}(t, x),(t, x) \in \mathbb{R}^{d+1}$, space-time white noise in $(\mathcal{S})^{*}$. From this we obtain a $d$-vector $\xi(t, x)$ of independent white noise fields for $t \in[0, T]$ :

$$
\xi(t, x)=\left(\dot{B}(t, x), \dot{B}\left(t+t_{0}, x\right), \ldots, \dot{B}\left(t+(d-1) t_{0}, x\right)\right)
$$


where $t_{0}>T$ is some fixed constant.

Consider the following Cauchy problem for $u \in C^{1,2}\left(D_{T}, \mathcal{S}^{*}\right)$, with $\alpha \in[0,1]$ fixed:

$$
\begin{aligned}
\frac{\partial u}{\partial t}(t, x)-L_{\alpha} u(t, x) & =\xi(t, x) \cdot \nabla u(t, x), \\
u(0, x) & =f(x) .
\end{aligned}
$$

Here $f$ belongs to $C_{b}^{2}\left(\mathbb{R}^{d}\right)$, the space of twice continuously differentiable functions with bounded derivatives up to the second order. Multiplication with $\xi$ is understood in the sense of Hitsuda-Skorokhod (the natural generalization of Itô's convention).

In [PVW] the authors prove that $(2.1)$ has a unique weak solution $u(t, x)$ in $(\mathcal{S})^{*}$, when $L_{\alpha}$ is uniformly elliptic and has coefficients in $C_{b}^{2}\left(\mathbb{R}^{d+1}\right)$. A weak $C^{1,2}$-function $u$ is a mapping $u: D_{T} \rightarrow(\mathcal{S})^{*}$ satisfying $\langle u(\cdot, \cdot), \varphi\rangle \in C^{1,2}\left(D_{T}\right)$ for every test function $\varphi \in(\mathcal{S})$, and the mapping

$$
\varphi \mapsto \partial_{i}\langle u(t, x), \varphi\rangle
$$

defines an element in $(\mathcal{S})^{*}$, the weak $x^{i}$-derivative of $u$, which is also denoted by $\partial_{i} u(t, x)$ (similarly for higher order derivatives). Clearly, every strong solution of (2.1) is also a weak solution, but the converse need not be true in general.

We apply the $S$-transformation to $(2.1)$ at $h \in \mathcal{S}\left(\mathbb{R}^{d+1}\right)$, i.e., we consider the dual pairing of (2.1) with the (normalized) test function $\varphi_{h}=: \exp \langle\cdot, h\rangle:=\exp \left\{-\frac{1}{2}|h|_{2}^{2}\right\} \exp \langle\cdot, h\rangle$, where $|h|_{2}$ denotes the norm in $L^{2}\left(\mathbb{R}^{d+1}\right)$. This yields

$$
\begin{aligned}
\frac{\partial v}{\partial t}(t, x ; h)-L_{\alpha} v(t, x ; h) & =h(t, x) \cdot \nabla v(t, x ; h), \\
v(0, x ; h) & =f(x)
\end{aligned}
$$

with $v(t, x ; h):=S(u(t, x))(h)$, and we denote by the same symbol $h$ the $d$-vector with components $h_{i}(t, x)=h\left(t+(i-1) t_{0}, x\right)$. To prove the stability result we will represent the solution $v_{\alpha}$ of this (non-stochastic) partial differential equation in terms of a stochastic process $X_{s}^{\alpha}$ with generator $L_{\alpha}$. We remark that this process is not related to the former $\xi(t, x)$; it just serves as a technical device.

Let the elliptic differential operator $L_{\alpha}$ be defined by

$$
L_{\alpha} u(t, x)=\frac{1}{2} \sum_{i, j=1}^{d} a_{\alpha}^{i j}(t, x) \frac{\partial^{2} u}{\partial x^{i} \partial x^{j}}(t, x)+\sum_{i=1}^{d} b_{\alpha}^{i}(t, x) \frac{\partial u}{\partial x^{i}}(t, x),
$$

where $b_{\alpha}(t, x)$ is a continuous $d$-vector and $a_{\alpha}(t, x)$ is a positive definite, continuous $d \times d-$ matrix which has a positive square-root $\sigma_{\alpha}(t, x)$. We suppose that $b_{\alpha}, \sigma_{\alpha}$, and the inverse matrix $\sigma_{\alpha}^{-1}$ (all denoted by the symbol $\rho_{\alpha}$ ) satisfy

$$
\left|\rho_{\alpha}(t, x)\right| \leq K(1+|x|), \quad\left|\rho_{\alpha}(t, x)-\rho_{\alpha}(t, y)\right| \leq K|x-y|,
$$

for all $(t, x) \in D_{T}$, where $K$ is a constant independent of $\alpha \in[0,1]$, and also that

$$
\lim _{\alpha \rightarrow 0}\left\{\sup _{|x| \leq N}\left|\rho_{\alpha}(t, x)-\rho_{0}(t, x)\right|\right\}=0,
$$


for all $N>0$ and $0 \leq t \leq T$. As is well-known, condition (C1) for $b_{\alpha}$ and $\sigma_{\alpha}$ implies that the ordinary stochastic differential equation $\left(B_{s}\right.$ denotes a $d$-dimensional Brownian motion on some probability space $(\Omega, \mathcal{F}, P)$ )

$$
d X_{s}^{\alpha}=\sigma_{\alpha}\left(t-s, X_{s}^{\alpha}\right) d B_{s}+b_{\alpha}\left(t-s, X_{s}^{\alpha}\right) d s, \quad X_{0}^{\alpha}=x,
$$

has a unique solution $\left\{X_{s}^{\alpha}\right\}_{0 \leq s \leq t}$. We remark that $t \in[0, T]$ is a fixed parameter in this equation and we often suppress the $t$ - and $x$-dependence in the notation of $X_{s}^{\alpha}$. As an easy consequence of $(\mathrm{C} 2)$ and Gronwall's lemma the following uniform continuity property holds, cf. [Fri, p. 119]:

$$
\lim _{\alpha \rightarrow 0}\left\{\sup _{(s, x) \in D_{t}} \mathbb{E}\left[\left(X_{s}^{\alpha}(x)-X_{s}^{0}(x)\right)^{2}\right]\right\}=0
$$

where $\mathbb{E}$ denotes the expectation with respect to $P$.

\section{The stability result}

We represent the solution $v_{\alpha}$ of $(2,2)$ in terms of $X_{s}^{\alpha}(x)$ (cf. [Fre]):

$$
v_{\alpha}(t, x ; h)=\mathbb{E}\left[f\left(X_{t}^{\alpha}\right) e^{\int_{0}^{t} \phi_{\alpha}\left(t-s, X_{s}^{\alpha}\right) d B_{s}-\frac{1}{2} \int_{0}^{t} \phi_{\alpha}^{2}\left(t-s, X_{s}^{\alpha}\right) d s}\right]
$$

where $\phi_{\alpha}(t, x):=\sigma_{\alpha}^{-1}(t, x) h(t, x)$ for all $(t, x) \in D_{T}$. Because $h$ is rapidly decreasing and $\sigma_{\alpha}^{-1}$ satisfies $(\mathrm{C} 1, \mathrm{C} 2)$ it is easy to verify:

$$
\begin{aligned}
\sup \left\{\left|\phi_{\alpha}(t, x)\right|:(\alpha, t, x) \in[0,1] \times D_{T}\right\} & =: R<\infty, \text { and } \\
\lim _{\alpha \rightarrow 0}\left\{\sup _{x \in \mathbb{R}^{d}}\left|\phi_{\alpha}(t, x)-\phi_{0}(t, x)\right|\right\} & =0
\end{aligned}
$$

for all $t \in[0, T]$. We are now prepared for the main theorem. Conditions (C1) and (C2) will be sufficient to prove stability of $u_{\alpha}$. Because these conditions are weaker than those which guarantee existence and uniqueness of $u_{\alpha}$ we shall assume existence and uniqueness.

Theorem 3.1 Let $L_{\alpha}$, given by (2.3), be such that (C1) and (C2) hold. Let $f \in C_{b}\left(\mathbb{R}^{d}\right)$ satisfy $|f(x)-f(y)| \leq l_{0}|x-y|$ for all $x, y \in \mathbb{R}^{d}$ with $l_{0} \geq 0$. Assume further that (2.1) has a unique solution $u_{\alpha} \in C^{1,2}\left(D_{T}, S^{*}\right)$ for each $\alpha \in[0,1]$. Then $u_{\alpha}(t, x)$ converges strongly to $u_{0}(t, x)$ as $\alpha \rightarrow 0$, uniformly in $D_{T}$.

Proof: In view of Lemma A.1 from the appendix it suffices to prove:

$$
\begin{aligned}
\left|v_{\alpha}(t, x ; z h)\right| & \leq K_{1} e^{K_{2}|z|^{2}\|h\|_{\infty}^{2},} \text { for all }(\alpha, t, x) \text {, and } \\
\sup _{D_{T}}\left|v_{\alpha}(t, x ; h)-v_{0}(t, x ; h)\right| & \rightarrow 0 \text { as } \alpha \rightarrow 0, \text { for all } h \in \mathcal{S}\left(\mathbb{R}^{d+1}\right) .
\end{aligned}
$$


Part (i) has already been proved in [PVW], in case there is no parameter $\alpha$. However, since the constants in (C1) and (C2) are independent of $\alpha$ one obtains - by the same proof the estimate (i) uniformly in $\alpha$.

To prove (ii) we set $F_{\alpha}:=\int_{0}^{t} \phi_{\alpha}\left(t-s, X_{s}^{\alpha}\right) d B_{s}-\frac{1}{2} \int_{0}^{t} \phi_{\alpha}^{2}\left(t-s, X_{s}^{\alpha}\right) d s$ and estimate:

$$
\begin{aligned}
\left|v_{\alpha}(t, x ; h)-v_{0}(t, x ; h)\right|^{2} & =\left|\mathbb{E}\left[\left(f\left(X_{t}^{\alpha}\right)-f\left(X_{t}^{0}\right)\right) e^{F_{0}}\right]+\mathbb{E}\left[f\left(X_{t}^{\alpha}\right)\left(e^{F_{\alpha}}-e^{F_{0}}\right)\right]\right|^{2} \\
& \leq 2 \mathbb{E}\left[\left(f\left(X_{t}^{\alpha}\right)-f\left(X_{t}^{0}\right)\right)^{2}\right] \mathbb{E}\left[e^{2 F_{0}}\right]+2 \mathbb{E}\left[f\left(X_{t}^{\alpha}\right)\left(e^{F_{\alpha}}-e^{F_{0}}\right)\right]^{2} \\
& \leq 2 M l_{0}^{2} \mathbb{E}\left[\left(X_{t}^{\alpha}-X_{t}^{0}\right)^{2}\right]+2\|f\|_{\infty}^{2} \mathbb{E}\left[\left|e^{F_{\alpha}}-e^{F_{0}}\right|\right]^{2}
\end{aligned}
$$

The last estimate follows from Lipschitz continuity of $f$ and from the following remark: When $\left(f_{s}\right)_{s \in[0, T]}$ is a continuous, adapted (to Brownian motion) process which is uniformly bounded for all $s$ and $\omega$, we find in [Fri, p. 152], that

$$
\mathbb{E}\left[e^{\int_{0}^{t} f_{s}(\omega) d B_{s}(\omega)-\frac{1}{2} \int_{0}^{t} f_{s}^{2}(\omega) d s}\right]=1, \quad \text { for all } t \in[0, T]
$$

From this it easily follows that for all $\lambda, \nu \in[0,2]$ there is a constant $c$, independent of $\lambda, \nu, \alpha$ and $t$, such that

$$
\mathbb{E}\left[e^{\lambda F_{\alpha}+\nu F_{0}}\right] \leq e^{c t} \leq e^{c T}=: M
$$

The first term in (3.2) converges to zero by (2.4), uniformly in $D_{T}$. The second term in (3.2) can be estimated as follows:

$$
\begin{aligned}
\mathbb{E}\left[\left|e^{F_{\alpha}}-e^{F_{0}}\right|\right]^{2} & =\mathbb{E}\left[\left|\int_{0}^{1} \frac{d}{d \lambda} e^{\lambda F_{\alpha}+(1-\lambda) F_{0}} d \lambda\right|\right]^{2} \\
& \leq \int_{0}^{1} \mathbb{E}\left[\left|F_{\alpha}-F_{0}\right| e^{\lambda F_{\alpha}+(1-\lambda) F_{0}}\right]^{2} d \lambda \\
& \leq \int_{0}^{1} \mathbb{E}\left[\left(F_{\alpha}-F_{0}\right)^{2}\right] \mathbb{E}\left[e^{2 \lambda F_{\alpha}+2(1-\lambda) F_{0}}\right] d \lambda \\
& \leq M \mathbb{E}\left[\left(F_{\alpha}-F_{0}\right)^{2}\right]
\end{aligned}
$$

Now we use $F_{\alpha}-F_{0}=\int_{0}^{t}\left(\phi_{\alpha}-\phi_{0}\right) d B_{s}-\frac{1}{2} \int_{0}^{t}\left(\phi_{\alpha}^{2}-\phi_{0}^{2}\right) d s$, the Itô isometry and (3.1):

$$
\begin{aligned}
\mathbb{E}\left[\left(F_{\alpha}-F_{0}\right)^{2}\right] & \leq 2 \mathbb{E}\left[\left(\int_{0}^{t}\left(\phi_{\alpha}-\phi_{0}\right) d B_{s}\right)^{2}\right]+2 \mathbb{E}\left[\left(\frac{1}{2} \int_{0}^{t}\left(\phi_{\alpha}^{2}-\phi_{0}^{2}\right) d s\right)^{2}\right] \\
& \leq 2 \mathbb{E}\left[\int_{0}^{t}\left(\phi_{\alpha}-\phi_{0}\right)^{2} d s\right]+2 \mathbb{E}\left[\frac{t}{4} \int_{0}^{t}\left(\phi_{\alpha}-\phi_{0}\right)^{2}\left(\phi_{\alpha}+\phi_{0}\right)^{2} d s\right] \\
& \leq 2\left(1+R^{2} T\right) \mathbb{E}\left[\int_{0}^{t} \sup _{x \in \mathbb{R}^{d}}\left|\phi_{\alpha}(t-s, x)-\phi_{0}(t-s, x)\right|^{2} d s\right] .
\end{aligned}
$$

In view of (3.1) and the dominated convergence theorem this completes the proof. 


\section{The zero diffusion limit}

In applications of stochastic differential equations one often considers the noise term in the sense of Stratonovich. In order to apply our stability result to this case the noise $\eta(t, x)$ - instead of $\xi(t, x)$ in $(2.1)$ - has to be sufficiently regular with respect to the $x$-variable. More precisely, let $\eta_{i}(t, x) \in(\mathcal{S})^{*}, i=1, \ldots, d$, be defined by

$$
\left\langle\eta_{i}(t, x), \varphi\right\rangle:=\int_{\mathbb{R}^{d}} \sum_{j=1}^{d} g_{i j}(x-u)\left\langle\xi_{j}(t, u), \varphi\right\rangle d u, \quad \varphi \in(\mathcal{S}),
$$

with non-zero functions $g_{i j} \in \mathcal{S}\left(\mathbb{R}^{d}\right)$.

Corollary 4.1 Substitute the noise $\xi$ in (2.1) by the smoothed noise (4.1) and assume that the resulting equation satisfies the conditions in Theorem 3.1. Then $u_{\alpha}(t, x)$ converges strongly to $u_{0}(t, x)$ as $\alpha \rightarrow 0$, uniformly in $D_{T}$.

Proof: As in the proof of Theorem 3.1 we show that (i) and (ii) hold for the modified equation. The $S$-transform of $\eta_{i}(t, x)$ reads:

$$
\tilde{h}_{i}(t, x ; h)=\int_{\mathbb{R}^{d}} \sum_{j=1}^{d} g_{i j}(x-u) h_{j}(t, u) d u
$$

and we observe: $\tilde{h}_{i}(\cdot, \cdot ; h) \in \mathcal{S}\left(\mathbb{R}^{d+1}\right)$. Instead of equation (2.2) we get

$$
\begin{aligned}
\frac{\partial v}{\partial t}(t, x ; h)-L_{\alpha} v(t, x ; h) & =\tilde{h}(t, x ; h) \cdot \nabla v(t, x ; h) \\
v(0, x ; h) & =f(x)
\end{aligned}
$$

and instead of (i) we obtain

$$
\left|v_{\alpha}(t, x ; z h)\right| \leq K_{1} e^{K_{2}|z|^{2}\|\tilde{h}\|_{\infty}^{2}} \text { for all }(\alpha, t, x)
$$

Now (i) follows from

$$
\|\tilde{h}\|_{\infty} \leq\|h\|_{\infty} \cdot \sum_{i, j=1}^{d}\left\|g_{i j}\right\|_{L^{1}\left(\mathbb{R}^{d}\right)}
$$

Since $\tilde{h}(\cdot, ; ; h)$ is rapidly decreasing $\tilde{\phi}_{\alpha}^{-1}(t, x)=\sigma_{\alpha}^{-1}(t, x) \tilde{h}(t, x ; h)$ also satisfies (3.1). This implies that part (ii) of the proof of Theorem 3.1 holds without any changes when $\phi_{\alpha}$ is replaced by $\tilde{\phi}_{\alpha}$.

Remark: The existence and uniqueness proof for solutions of (2.1) given in [PVW] easily extends to the case where $\xi$ is substituted by the (smoothed) noise $\eta$. The necessary modifications are similar to those in the above proof. 
Informally, the covariance of $\eta$ reads

$$
\mathbb{E}_{\mu}\left[\eta_{i}(t, x) \eta_{j}(s, y)\right]=\delta(t-s) K_{i j}(x, y)
$$

where $\mathbb{E}_{\mu}$ is expectation with respect to the white noise measure $\mu$, and

$$
K_{i j}(x, y)=\sum_{r=1}^{d} \int_{\mathbb{R}^{d}} g_{i r}(x-u) g_{j r}(y-u) d u
$$

Notice that $K_{i j}:=K_{i j}(x, x)$ are constant elements of a symmetric $d \times d$-matrix $K$. We are now prepared to consider the Cauchy problem in Stratonovich sense (denoted by $\circ$ ):

$$
\frac{\partial u}{\partial t}-L_{\alpha} u=\eta \circ \nabla u, \quad u(0, x)=f(x)
$$

Rewritten as an Itô equation this gives (cf. [Ku]):

$$
\begin{gathered}
\frac{\partial u}{\partial t}-L_{\alpha} u-\frac{1}{2} \sum_{i, j=1}^{d} K_{i j} \frac{\partial^{2} u}{\partial x^{i} \partial x^{j}}-\left.\frac{1}{2} \sum_{i, j=1}^{d} \frac{\partial K_{i j}(x, y)}{\partial x^{i}}\right|_{y=x} \frac{\partial u}{\partial x^{j}}=\eta \nabla u \\
u(0, x)=f(x) .
\end{gathered}
$$

When the coefficients of $L_{\alpha}$ tend uniformly to zero, the second order terms of (4.3) tend uniformly to $\frac{1}{2} \sum K_{i j} \partial_{i} \partial_{j} u$. The combination of this with Corollary 4.1 and the above remark yields:

Corollary 4.2 Let $L_{\alpha}, \alpha \in[0,1]$, be uniformly elliptic operators with coefficients in $C_{b}^{2}\left(D_{T}\right)$ such that $(C 1, C 2)$ hold with $b_{0}=0$ and $\sigma_{0}=0$. Assume further that $\eta$ is given by (4.1) such that $K$ is positive definite, and the inverse of $\sigma_{\alpha}+K$ satisfies (C1,C2). Then (4.2) has a unique solution $u_{\alpha} \in C^{1,2}\left(D_{T}, \mathcal{S}^{*}\right)$ which converges strongly to $u_{0}$ as $\alpha \rightarrow 0$, uniformly in $D_{T}$, and $u_{0}$ satisfies

$$
\frac{\partial u}{\partial t}=\eta \circ \nabla u, \quad u(0, x)=f(x)
$$

\section{Appendix}

The $S$-transformation is an important tool in white noise analysis. One of its applications is to solve differential equations by solving the corresponding $S$-transformed equations. A basic question is then how the inverse $S$-transformation behaves under limits with respect to parameters $x \in \mathbb{R}^{n}$, like continuity and/or differentiability with respect to $x$. There are several notions of differentiability in the literature, like weak differentiability, or differentiability of coefficients in chaos or Hermite expansions. In this appendix we derive 
a sufficient condition for when the limit $\lim _{x \rightarrow x_{0}} S^{-1} F(x)$ exists with respect to the strong topology on $(\mathcal{S})^{*}$. We remark that the resulting notion of strong differentiability is stronger than each of the before mentioned notions.

Consider the $S$-transform of $\Phi \in(\mathcal{S})^{*}$ evaluated at $h \in \mathcal{S}\left(\mathbb{R}^{n}\right)$ :

$$
F(h):=S \Phi(h)=\left\langle\Phi,: e^{\langle\cdot, h\rangle}:\right\rangle
$$

The space of mappings $F: \mathcal{S}\left(\mathbb{R}^{d}\right) \rightarrow \mathbb{R}$ obtained in this way is denoted by $\mathcal{U}$. Since $S$ is injective the correspondence between $(\mathcal{S})^{*}$ and $\mathcal{U}$ is one-to-one.

Lemma A.1 Let $X$ be a first countable topological space, $Y$ be an arbitrary set, and $F$ : $X \times Y \rightarrow \mathcal{U}$ be such that, for every $h \in \mathcal{S}\left(\mathbb{R}^{n}\right)$ :

(i) The function $F(\cdot, y ; h): X \rightarrow \mathbb{R}, F(x, y ; h):=F(x, y)(h)$ is continuous at $x_{0}$, uniformly with respect to $y$, i.e.

$$
\sup _{y \in Y}\left|F(x, y ; h)-F\left(x_{0}, y ; h\right)\right| \rightarrow 0, \text { as } x \rightarrow x_{0} .
$$

(ii) There exists a continuous seminorm $\|\cdot\|$ on $\mathcal{S}\left(\mathbb{R}^{n}\right)$ and $K>0$, such that

$$
|F(x, y ; z h)| \leq K e^{|z|^{2}\|h\|^{2}}
$$

for all $(x, y) \in X \times Y$ and all $z \in \mathbb{C}$.

Then there exists $r \in \mathbb{N}$ such that $\Phi_{y}(x):=S^{-1}(F(x, y)) \in(\mathcal{S})_{-r}$, for all $(x, y) \in X \times Y$. The mapping $x \mapsto \Phi_{y}(x)$ is continuous at $x_{0}$, uniformly with respect to $Y$ :

$$
\sup _{y \in Y}\left\|\Phi_{y}(x)-\Phi_{y}\left(x_{0}\right)\right\|_{-r} \rightarrow 0 \quad \text { as } \quad x \rightarrow x_{0} .
$$

Consequently $\Phi_{y}$, considered as a mapping from $X$ into $(\mathcal{S})^{*}$, is strongly continuous at $x_{0}$, uniformly with respect to $Y$.

Proof: We need two facts from [PS], [KLPSW]: Firstly, (ii) implies there exists $C \geq 0$ and $q \geq 0$ such that $\Phi_{y}(x) \in(\mathcal{S})_{-q}$ and $\left\|\Phi_{y}(x)\right\|_{-q} \leq C$ for all $(x, y) \in X \times Y$. Secondly, the vector space $\mathcal{E} \subset(\mathcal{S})_{-q}$ generated by $\left\{\exp \langle\cdot, h\rangle \mid h \in \mathcal{S}\left(\mathbb{R}^{n}\right)\right\}$ is dense in $(\mathcal{S})_{-q}$.

Since $X$ is first countable it suffices to consider sequences. Let $\left(x_{n}\right)$ be a sequence in $X$ which converges to $x_{0}$. From (i) follows

$$
\sup _{y \in Y}\left|\left(\Phi_{y}\left(x_{n}\right), \varphi\right)_{-q}-\left(\Phi_{y}\left(x_{0}\right), \varphi\right)_{-q}\right| \rightarrow 0 \quad \text { as } n \rightarrow \infty
$$

for every $\varphi \in \mathcal{E}$. Since $\Phi_{y}\left(x_{n}\right)$ is bounded in $(\mathcal{S})_{-q}$ we find that (A.1) holds for all $\varphi \in$ $(\mathcal{S})_{-q}$.

Let $\left\{e_{1}, e_{2}, \ldots\right\}$ be a CONS in $(\mathcal{S})_{-q}$ and consider the orthogonal expansion

$$
\Phi_{y}\left(x_{n}\right)-\Phi_{y}\left(x_{0}\right)=\sum_{k=1}^{\infty}\left(a_{k}^{(n)}(y)-a_{k}^{(0)}(y)\right) e_{k}
$$


where

$$
\begin{aligned}
\left|a_{k}^{(n)}(y)-a_{k}^{(0)}(y)\right| & =\left|\left(\Phi_{y}\left(x_{n}\right), e_{k}\right)_{-q}-\left(\Phi_{y}\left(x_{0}\right), e_{k}\right)_{-q}\right| \\
& \leq\left\|\Phi_{y}\left(x_{n}\right)-\Phi_{y}\left(x_{0}\right)\right\|_{-q} \leq 2 C .
\end{aligned}
$$

Now (A.1) implies that for each $k \in \mathbb{N}$

$$
\sup _{y \in Y}\left|a_{k}^{(n)}(y)-a_{k}^{(0)}(y)\right| \rightarrow 0, \text { as } n \rightarrow \infty
$$

The identity mapping $i:(\mathcal{S})_{-q} \hookrightarrow(\mathcal{S})_{-r}, r=q+1$, is a Hilbert-Schmidt operator, so that

$$
\sum_{k=1}^{\infty}\left\|e_{k}\right\|_{-r}^{2}=\sum_{k=1}^{\infty}\left\|i\left(e_{k}\right)\right\|_{-r}^{2}=\sum_{k=1}^{\infty} N_{k}^{2}=: M<\infty .
$$

This implies that for any $\epsilon>0$ there exists $m=m(\epsilon)$ such that $(2 C)^{2} \sum_{k=m}^{\infty} N_{k}^{2}<\epsilon / 2$. Using this and (A.2) we estimate as follows:

$$
\begin{aligned}
\left\|\Phi_{y}\left(x_{n}\right)-\Phi_{y}\left(x_{0}\right)\right\|_{-r}^{2} & =\sum_{k=1}^{\infty}\left|a_{k}^{(n)}(y)-a_{k}^{(0)}(y)\right|^{2} N_{k}^{2} \\
& \leq \sum_{k=1}^{m-1}\left|a_{k}^{(n)}(y)-a_{k}^{(0)}(y)\right|^{2} N_{k}^{2}+(2 C)^{2} \sum_{k=m}^{\infty} N_{k}^{2} \\
& \leq \frac{\epsilon}{2}+\frac{\epsilon}{2} \text { for all } y \in Y \text { and all } n \geq n_{0} .
\end{aligned}
$$

This shows that the mapping $\Phi_{y}$ from $X$ into $(\mathcal{S})_{-r}$ is continuous, uniformly with respect to $Y$. Composition of $\Phi_{y}$ with the strongly continuous embedding $(\mathcal{S})_{-r} \hookrightarrow(\mathcal{S})^{*}$ completes the proof.

We can now give a criterion for when a weak solution of (2.1) is a strong solution.

Corollary A.2 Let $u$ be a weak solution of (2.1), $v(t, x ; h):=S(u(t, x))(h)$, and suppose the $t$-derivative of $v$ and the $x$-derivatives of $v$ up to the second order satisfy (ii). Then $u$ is a strong solution of (2.1).

Proof: We first show that the derivatives in (2.1) exist for each fixed $(t, x) \in D_{T}$ in the strong sense. Let $b_{i} \in \mathbb{R}^{d}$ be the unit vector in the $\mathrm{i}$-th coordinate direction and consider

$$
\Phi_{i}(\epsilon):=\frac{u\left(t, x+\epsilon b_{i}\right)-u(t, x)}{\epsilon}, \quad \epsilon \neq 0 .
$$

The $S$-transform is: $\left(S \Phi_{i}(\epsilon)\right)(h)=: F(\epsilon, i ; h)=\left[v\left(t, x+\epsilon b_{i} ; h\right)-v(t, x ; h)\right] / \epsilon$. Since $v(\cdot, \cdot ; h)$ is differentiable $F(\epsilon, i ; h)$ converges to $F(0, i ; h):=\partial_{i} v(t, x ; h)$ as $\epsilon \rightarrow 0$. Thus, $F$ satisfies (i) in Lemma A.1, with $X:=\mathbb{R}$ and $Y:=\{1, \ldots, d\}$. Also (ii) holds:

$$
|F(\epsilon, i ; z h)| \leq \frac{1}{|\epsilon|} \int_{0}^{\epsilon}\left|\frac{\partial v}{\partial x_{i}}\left(t, x+s b_{i} ; z h\right)\right| d s \leq \frac{1}{|\epsilon|} \int_{0}^{\epsilon} K e^{|z|^{2}\|h\|^{2}} d s=K e^{|z|^{2}\|h\|^{2}}
$$


By Lemma A.1 $\Phi_{i}(\epsilon)$ converges to $\Phi_{i}(0):=S^{-1} \partial_{i} v(t, x ; \cdot)$ strongly in $(S)^{*}$ as $\epsilon \rightarrow 0$, which means by definition that $u$ is strongly differentiable with respect to $x_{i}$. (Of course the same holds for the strong $t$-derivative of $u$.) Moreover it follows that the weak derivatives of $u$ coincide with the strong derivatives. But then the same method of proof shows that the strong second order $x$-derivatives of $u$ exist as well.

By assumption (2.1) holds in the weak sense. Since weak and strong derivatives coincide (2.1) also holds in the strong sense.

Finally $u, \partial_{i} u$ etc. depend continuously on $(t, x) \in D_{T}$. This follows from Lemma A.1 for the choice $X:=D_{T}$ (no $y$-dependence) and the observation that (i) and (ii) hold for $v(t, x ; h)$ and for the derivatives up to the second order.

\section{References.}

[Ch] P.L. Chow: Generalized Solution of some Parabolic Equations with a Random Drift, J. Appl. Math. Optimization 20, pp. 81-96 (1989).

[DP] T. Deck and J. Potthoff: On A Class Of Stochastic Partial Differential Equations Related To Turbulent Transport. Preprint Nr.189/95, University of Mannheim, Germany.

[Fri] A. Friedman: Stochastic differential equations and applications, Academic Press (1975).

[Fre] Freidlin, Functional Integration and Partial Differential Equations, Princeton University Press (1985).

[KLPSW] Y. Kondratiev, P. Leukert, J. Potthoff, L. Streit and W. Westerkamp, Generalized Functionals in Gaussian spaces - The characterization theorem revisited, Preprint Nr. 175/94, Mannheim University, 1994, to appear in J. Func. Anal.

[Ku] H. Kunita: Stochastic Flows and Stochastic Differential Equations. Cambridge: Cambridge Universit Press (1990).

[P] J. Potthoff, White Noise Approach to Parabolic Stochastic Partial Differential Equations in: Stochastic Analysis and Applications in Physics, A. I. Cardoso et al. (ed.'s). Kluwer Academic Publisher, Dordrecht (1994).

[PS] J. Potthoff and L. Streit: A Characterization of Hida Distributions, J. Funct. Anal. 101, pp. 212-229 (1991).

[PVW] J. Potthoff, G. Våge and H. Watanabe: Generalized Solutions of Linear Parabolic Stochastic Partial Differential Equations, Preprint Nr. 210/96, University of Mannheim, Germany. 